\title{
Rapid Photocatalytic Degradation of Pollutant from Water under UV and Sunlight via Cellulose Nanofiber Aerogel Wrapped by $\mathrm{TiO}_{2}$
}

\author{
Song Li $\mathbb{D},{ }^{1}$ Xiaofeng Hao, ${ }^{2}$ Xiangdong Dai $\mathbb{D},{ }^{1}$ and Tao Tao $\mathbb{D}^{2}$ \\ ${ }^{1}$ College of Furniture and Art Design, Central South, University of Forestry and Technology, Changsha 410004, China \\ ${ }^{2}$ Material Science and Engineering College, Central South, University of Forestry and Technology, Changsha 410004, China
}

Correspondence should be addressed to Xiangdong Dai; daixiangdong2000@163.com and Tao Tao; taotaohunter@163.com

Received 31 January 2018; Revised 7 April 2018; Accepted 16 April 2018; Published 17 July 2018

Academic Editor: Xin Jin

Copyright ( 2018 Song Li et al. This is an open access article distributed under the Creative Commons Attribution License, which permits unrestricted use, distribution, and reproduction in any medium, provided the original work is properly cited.

\begin{abstract}
Semiconductor photocatalysis can be used to decompose various organic compounds in open air under aqueous conditions with solar energy. A cellulose nanofiber/ $/ \mathrm{TiO}_{2}$ aerogel (CNFT) for rapid photocatalytic degradation of methylene blue (MB) solution was successfully fabricated through a facile hydrothermal method. $\mathrm{TiO}_{2}$ nanoparticles adhered to the surface of cellulose nanofibers as scaffold through a hydrogen bond and were well-distributed. The particle size distribution data showed that the mean diameter of $\mathrm{TiO}_{2}$ nanoparticles was around $6.8 \mathrm{~nm}$. In the system of the as-prepared CNFT, CNF played important roles in adsorption of pollutant and in stopping the electron hole derived from $\mathrm{TiO}_{2}$ from recombining. The composite aerogel exhibited efficient photocatalytic activity in the degradation of pollutant (MB) under ultraviolet light (UV) and sunlight irradiation. The composite aerogel could be regarded as a green and portable photocatalyst, because it was promising for wastewater treatment application as a result of its good high photocatalytic activity and stability under ultraviolet light and sunlight irradiation.
\end{abstract}

\section{Introduction}

With the urgency of water pollution, much attention was paid to the treatment of water pollution. Nowadays, the most commonly purified systems were employing filters packed with adsorbents like activated carbon, which do not constitute a definite conversion solution, merely transferring the pollutant to a different phase, thus requiring periodic maintenance and producing considerable amounts of waste. To prevent health hazard effects of water pollutions, urgent and new countermeasures must be implemented. The development of an efficient, green, and low-cost method for the removal of water pollutant compounds was essential. Photocatalysis continues to develop as a promising alternative technology for the renewal of our water system.

With its high photoreactivity, biological and chemical inertness, cost effectiveness, nontoxicity, and long-term stability against corrosion, $\mathrm{TiO}_{2}$ was widely used as a photocatalyst in waterwater treatment. [1]. Many organic pollutants, such as alcohols [2], phenols [3], dye [4], and carboxylic acids [5], can be photodegraded by $\mathrm{TiO}_{2}$ nanoparticles under ultraviolet light (UV) or visible light irradiation. In the process of degradation, oxidation reactions allow the destruction of organic pollutants mainly through reactions with reactive oxygen species, such as hydroxyl radicals [6], which turn to be $\mathrm{CO}_{2}$ and $\mathrm{H}_{2} \mathrm{O}$. The main photocatalytic procedure was the creation of electron-hole pairs interacting with the radiation, which were subsequently separated to be utilized in reduction and oxidation steps based on $\mathrm{TiO}_{2}$ catalysts [7]. However, the practical applications of $\mathrm{TiO}_{2}$ were still challenging in terms of their poor photocatalytic efficiency due to unwanted recombination of the electron-hole pairs [8]. In order to increase the photocatalytic activity, many researches had been conducted, for example, doping with nonmetallic atoms (namely $\mathrm{S}, \mathrm{N}, \mathrm{P}$, etc.) [9], transition metals (namely, Pt, 
$\mathrm{Cu}, \mathrm{Mg}$, etc.) [10], and polymers [11], or codoping with several ions.

Aerogels are highly porous materials that can have extremely low densities, large open pores, and low thermal transport, which can be applied in multiple fields such as catalysis [12, 13], superadsorbents, and desalination [13]. Most aerogels are fabricated from silica, metal oxides, pyrolyzed organic polymers, and carbon-based materials [14]. However, the traditional aerogels suffer from poor mechanical robustness, which has led to numerous attempts to overcome this problem. Several nanomaterials including carbon nanotubes $[15,16]$, carbonaceous nanofibers [17], and graphene $[18,19]$ have been recently developed as building blocks in the assembly of strong aerogels. Among them, cellulose nanofiber (CNF) from natural plants has attracted considerable attention because of its low density, high specific area, low thermal conductivity, and low dielectric permittivity [20]. CNF could be isolated from biomass materials [21]. The long and entangled CNF of cellulose I crystal type can form a strong aerogel network because of its fibrillar morphology and strong mutual hydrogen bonds, which facilitate mechanical ductility and flexibility of the aerogels $[22,23]$. Potential applications of nanometer-sized cellulose included tissue engineering [24], efficient catalysts [25], electrooptical films [26], biodegradable packaging materials [27], nanofiber-reinforced composites [28], gas-barrier films [29], cosmetics [30], and other hightech and high-performance materials. The research of inorganic nanoparticles/cellulose composite materials was a very exciting research area currently such as electrical [31], magnetic [32], and optical [33] properties. Because they have distinct size-dependent physicochemical and optoelectronic properties, the inorganic nanoparticles/cellulose composite materials could be considered as a candidate for the improvement of stability, retaining of the special morphology, and control of the growth of nanoparticles. The CNF aerogels have been used as templates for inorganic $\mathrm{MnO}_{2}$ nanosheets [34] and $\mathrm{Na}_{2} \mathrm{Ti}_{3} \mathrm{O}_{7}$ nanoparticles [35] and further functionalized to achieve ideal highly efficient water purification.

In this study, a cellulose nanofiber/ $\mathrm{TiO}_{2}(\mathrm{CNFT})$ aerogel for rapid photocatalytic degradation of methylene blue in water was successfully fabricated through a facile hydrothermal method. In the system of the CNFT composite, $\mathrm{TiO}_{2}$ nanoparticles could be adhered to wet CNF as a scaffold [36]. And the functionality of photodegradation could be enhanced through a synergistic interaction between the $\mathrm{CNF}$ and $\mathrm{TiO}_{2}$ nanoparticles due to interactive structures in materials.

\section{Materials and Methods}

2.1. Materials. The bamboo was obtained from the bamboo forest of Zhejiang A\&F University. Tetrabutyl titanate, titanium dioxide (P25), urea, citric acid, methylene blue, and other chemicals were of analytical grade. All the chemicals were used as received. Deionized water was used in all experiments.

2.2. Preparation of the CNFT Composite Aerogel. A CNF solution was prepared by the method of chemical pretreatment combined with ultrasonic treatment, and the CNF from bamboo was referred to Xiao et al [37] and used for characterization. $100 \mathrm{mg}$ CNF was put into $100 \mathrm{ml}$ deionized water, and then a few milliliters of tetrabutyl titanate, $0.6 \mathrm{~g}$ urea, and $4 \mathrm{~g}$ citric acid were added with magnetic stirring for $30 \mathrm{~min}$ at room temperature. Subsequently, the mixtures were transferred to a Teflon-lined stainless-steel autoclave. The autoclave was placed in an oven and heated at $180^{\circ} \mathrm{C}$ for $24 \mathrm{~h}$. The suspension was cooled down naturally and washed by a centrifuge treatment with $6000 \mathrm{rpm} / \mathrm{s}$ for $5 \mathrm{~min}$. To obtain the homogeneous solution, the bulk after centrifuge treatment was put into deionized water and then underwent an ultrasonic treatment for 1 min with an output power of $50 \mathrm{~W}$ in an ice water bath to disperse the solution. The obtained concentrated suspension, with a high viscosity and gel-like appearance, was freeze-dried at $-55^{\circ} \mathrm{C}$ at $10 \mu \mathrm{Pa}$ for $60 \mathrm{~h}$. The lightweight, solid, and sponge-like aerogel was produced. The volume of tetrabutyl titanate was $1 \mathrm{ml}, 2 \mathrm{ml}$, and $4 \mathrm{ml}$ and was coded as CNFT1, CNFT2, and CNFT4, respectively.

2.3. Characterization. The morphological feature of the CNF and CNFT composite was observed using a scanning electron microscope (SEM, FEI, Quanta 200, USA) and transmission electron microscope (TEM, FEI, Tecnai G20, USA). Crystalline structures were identified by $\mathrm{X}$-ray diffraction technique (XRD, Rigaku, D/Max 2200, Japan) operating with $\mathrm{Cu} \mathrm{K \alpha}$ radiation $(\lambda=1.5418 \AA)$ at a scan rate $(2 \theta)$ of $2^{\circ} \cdot \mathrm{min}^{-1}$ and the accelerating voltage of $40 \mathrm{kV}$ and the applied current of $30 \mathrm{~mA}$ ranging from $5^{\circ}$ to $90^{\circ}$. The X-ray photoelectron spectroscopy (XPS, ULVAC-PHI Inc., Japan) analysis of the specimen was carried out using a microfocused $(100 \mathrm{~lm}$, $25 \mathrm{~W}, 15 \mathrm{kV})$ monochromatic Al-Ka source $(\mathrm{hm}=1486.6 \mathrm{e}$ $\mathrm{V})$, a hemispherical analyzer, and a multichannel detector. Thermal-physical properties of the CNF and CNFT composite were simultaneously assessed with a Hot Disk TPS $2500 \mathrm{~S}$ instrument (Hot Disk AB) using $20 \mathrm{~mW}$ output power in the transient mode from $25^{\circ} \mathrm{C}$ to $800^{\circ} \mathrm{C}$, with a heating rate of $10^{\circ} \mathrm{C} \cdot \mathrm{min}^{-1}$ under nitrogen atmosphere. FTIR spectra were recorded on a Fourier transform infrared instrument (Magna 560, Nicolet, Thermo Electron Corp.) in the range of $400-4000 \mathrm{~cm}^{-1}$ with a resolution of $4 \mathrm{~cm}^{-1}$. UV-Vis spectra were recorded on a TU-9001 spectrophotometer equipped with a Labsphere diffuse reflectance accessory. The Brunauer-Emmett-Teller (BET) surface areas were measured by nitrogen adsorption isotherm measurements at $77 \mathrm{~K}$ on a JW-BK instrument.

2.4. Photocatalytic Activity. The photocatalytic activities of CNF, P25, $\mathrm{TiO}_{2}$ synthesized at similar conditions without adding cellulose nanofiber, and CNFT were measured by the photodegradation of methylene blue as model reaction under UV and sunlight illumination. Typically, six groups of $10 \mathrm{mg}$ of the prepared CNFT composites were dipped in $50 \mathrm{ml} \mathrm{MB}$ solution (15 ppm). The mixture solution was then exposed to UV irradiation produced by a tunable high-pressure $\mathrm{Hg}$ lamp with the main peak at a wavelength of $365 \mathrm{~nm} .10 \mathrm{ml}$ of suspension was extracted afterwards per $5 \mathrm{~min}$ from one of the six groups and then 


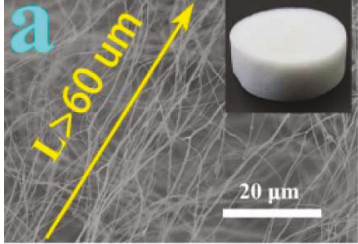

(a)

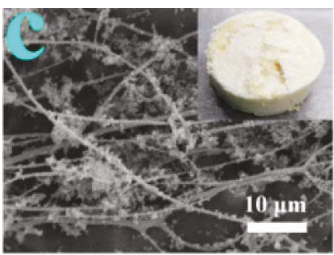

(c)

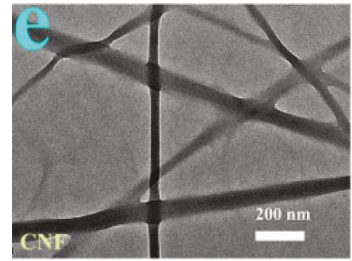

(e)

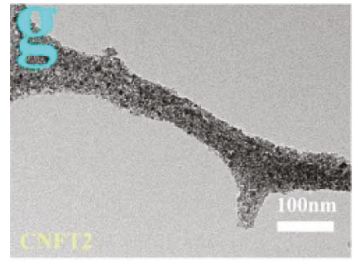

(g)

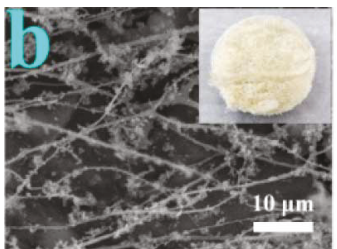

(b)

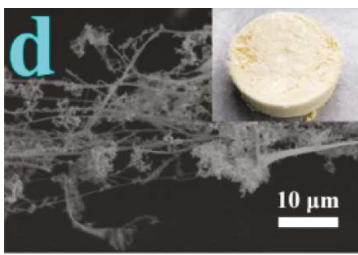

(d)

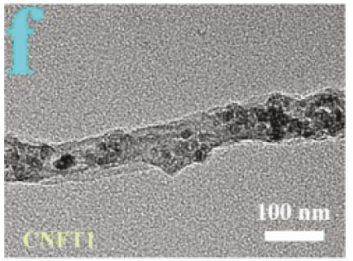

(f)

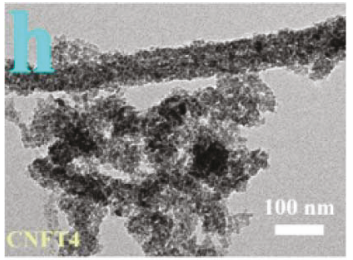

(h)

FIgURE 1: The morphologies of CNF and CNFT. (a)-(d) The SEM micrographs of CNF, CNFT1, CNFT2, and CNFT4, respectively, and the shape in the inset. (e)-(h) The TEM images of CNF, CNFT1, CNFT2, and CNFT4, respectively.

centrifuged at $6000 \mathrm{rpm}$ for $5 \mathrm{~min}$ to get rid of the CNFT from the supernatant. Samples were exposed to sunlight. The selected exposure time was $1 \mathrm{~h}$ every day from 1 p.m. to 2 p.m. on sunny days. Then, the solution was analyzed by recording the UV-Vis spectrum of $\mathrm{MB}$ at the maximum absorbance of $664 \mathrm{~nm}$. The degradation of $\mathrm{MB}$ was studied with UV visible spectroscopy measurement carried out on a UV visible spectrophotometer (model $\mathrm{Tu}-1901)$ in the range of $450-750 \mathrm{~nm}$. The normalized temporal concentration changes $\left(\mathrm{C} / \mathrm{C}_{0}\right)$ of $\mathrm{MB}$ during the photodegradation were proportional to the normalized maximum absorbance $\left(\mathrm{A} / \mathrm{A}_{0}\right)$ and derived from the changes in the dye's absorption profile $(664 \mathrm{~nm})$ at a given time interval. The photodegradation rate was calculated by the following equation:

$$
\text { Photodegradation rate }=\frac{\left(C_{0}-C\right)}{C_{0}} \times 100 \% \text {, }
$$

where $C$ is the concentration of $\mathrm{MB}$ solution at time and $C_{0}$ is the initial concentration.

\section{Results and Discussion}

Figure 1 shows the morphologies of CNF and CNFT. After water was completely removed through freeze-drying, a lightweight sponge-like aerogel was produced which did not have any collapse with the density of $1 \mathrm{mg} \cdot \mathrm{cm}^{-3}$. As shown in Figure 1(a), the macroscopic morphology of the CNF aerogel was white with integrity and had a well-defined shape. The SEM image showed the micromorphology image of the CNF fibrils; the length of CNF fibrils was much longer than $60 \mu \mathrm{m}$. From Figures 1(b)-1(d), it shows the SEM images of CNFT1, CNFT2, and CNFT4, which possessed an interconnected 3D network microstructure. The shapes are shown in the inset. They were turned to be beige from white CNF aerogel because of the formation of $\mathrm{TiO}_{2}$ nanoparticles in the samples inside. After modification by $\mathrm{TiO}_{2}$ nanoparticles, the structure of the CNFT2 composite showed filamentous fibers covered by nanoparticles. Figure 1(e) shows TEM images of CNF samples, which exhibited some slender fibrils with an average diameter of the CNF fibrils of about $63.2 \mathrm{~nm}$. The CNF exhibited a high draw ratio about 1000 according to the length referred before. The TEM images of 


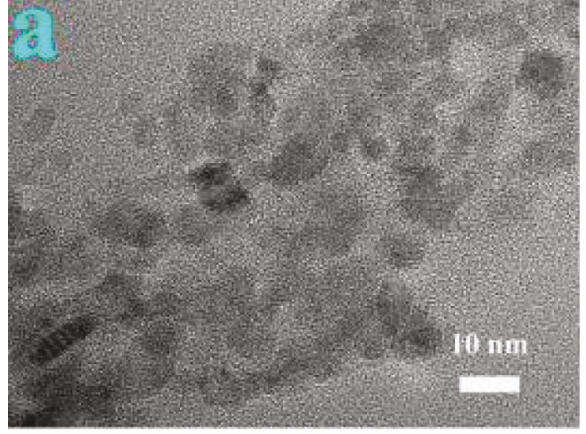

(a)

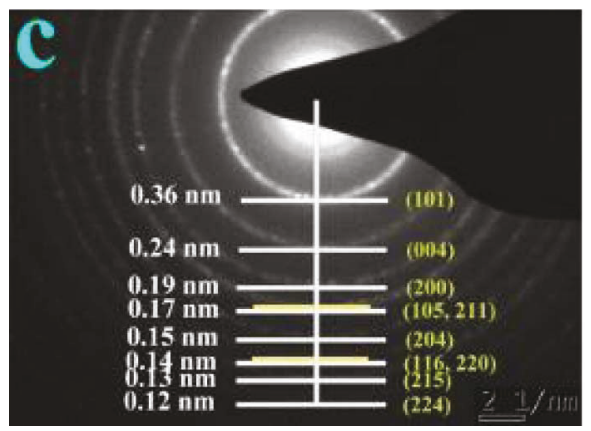

(c)

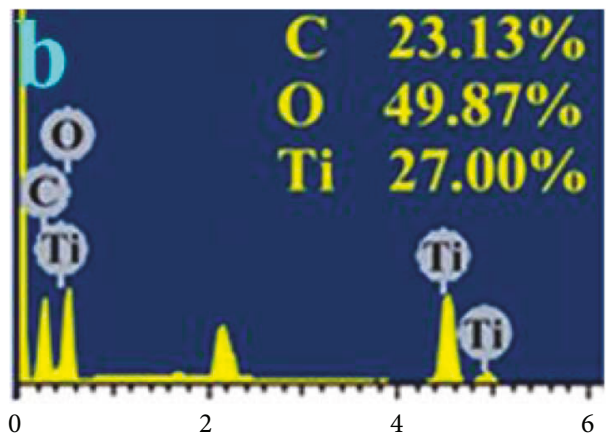

(b)

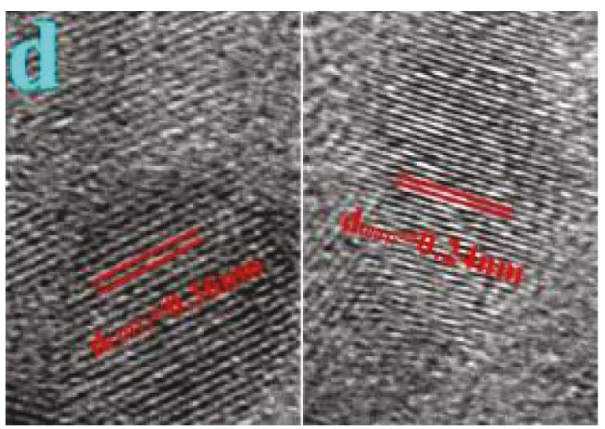

(d)

FIgure 2: (a) The enlarged view of the TME image, (b) the EDXA spectra, (c) selected area electron diffraction, and (d) high-resolution TEM image, of CNFT2.

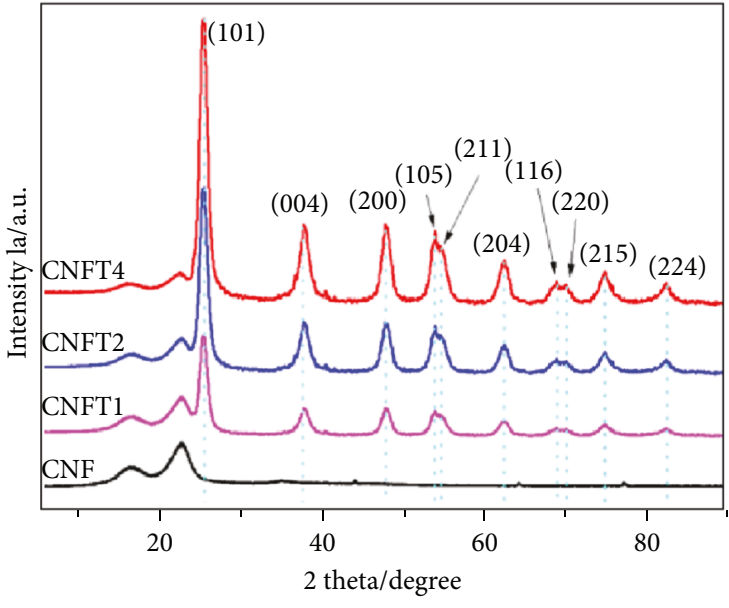

FIgURE 3: XRD patterns of (a) CNF and (b) CNFT.

samples CNFT1, CNFT2, and CNFT4 are shown in Figures 1(f)-1(h). The surface of CNFT4 was wrapped by $\mathrm{TiO}_{2}$ nanoparticles in the surface of fibers, which was denser than that of CNFT2. With more and more $\mathrm{TiO}_{2}$ nanoparticles formed, the CNF was not able to contain these particles so that some of $\mathrm{TiO}_{2}$ particles were outside of the fibers. In CNFT2, the TEM images of the sample showed that a large quantity of $\mathrm{TiO}_{2}$ nanoparticles were formed like a film structure and coated on the surface of the CNF fibrils. Furthermore, the $\mathrm{TiO}_{2}$ nanoparticles were well-distributed. In terms of CNFT1, $\mathrm{TiO}_{2}$ nanoparticles were interspersed basically in the surface of the cellulose fibers.
Figure 2(a) is the enlarged view of CNFT2; the particle size distribution data showed that the mean diameter of $\mathrm{TiO}_{2}$ nanoparticle sizes was around $6.8 \mathrm{~nm}$. The energydispersive spectrum (EDS) in Figure 2(b) indicates that there were $\mathrm{C}, \mathrm{O}$, and Ti elements in the composite sample, suggesting that the $\mathrm{TiO}_{2}$ nanoparticles had been synthesized in the composite aerogel. Figure 2(c) shows the SAED pattern, in which eight main diffraction rings correspond to the (101), (004), (200), (105, 211), (204), (116, 220), (215), and (224) planes of polycrystalline $\mathrm{TiO}_{2}$ with a spherical structure. Besides, the diffraction rings were discontinuous and consist of rather sharp spots, indicating that the nanoparticles were well crystallized. To further investigate the distribution of $\mathrm{TiO}_{2}$ particles, the HRTEM image was displayed as shown in Figure 2(d). The results clearly revealed a lattice fringe with a d-spacing of $0.36 \mathrm{~nm}$ and $0.24 \mathrm{~nm}$ corresponding to the (101) and (004) planes, which were present in anatase phase $\mathrm{TiO}_{2}$.

The phase structures of CNF and CNFT were revealed by XRD analysis. As shown in Figure 3, it was clear that diffraction peaks at $16.5^{\circ}(101)$ and $22.5^{\circ}(002)$ were typical cellulose peaks [38]. The CNF clearly retained the cellulose structure, indicating that the CNF was prepared successfully by isolating from the raw material bamboo. The diffraction peaks of CNFT composite samples were observed at $25.2^{\circ}, 37.7^{\circ}$, $47.8^{\circ}, 53.8^{\circ}, 55.0^{\circ}, 62.7^{\circ}, 68.7^{\circ}, 70.3^{\circ}, 74.9^{\circ}$, and $82.6^{\circ}$, which corresponded to planes (101), (004), (200), (105), (211), (204), (116), (220), (215), and (224) [39], respectively. It was clear that diffraction peaks in the XRD pattern were well indexed to the anatase phase of $\mathrm{TiO}_{2}$. The prepared $\mathrm{TiO}_{2}$ also 


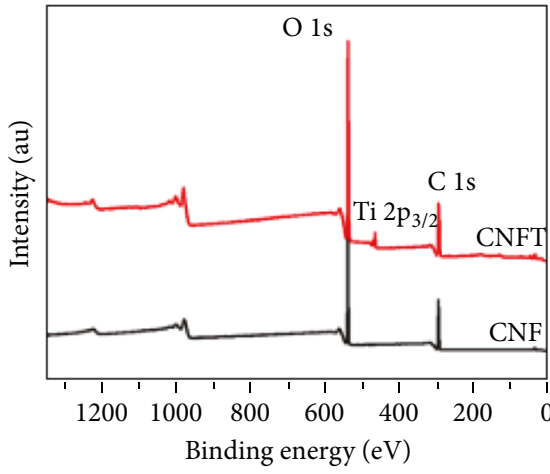

(a)

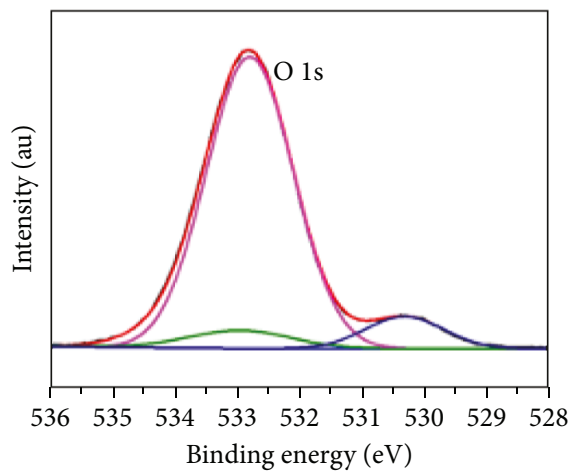

(c)

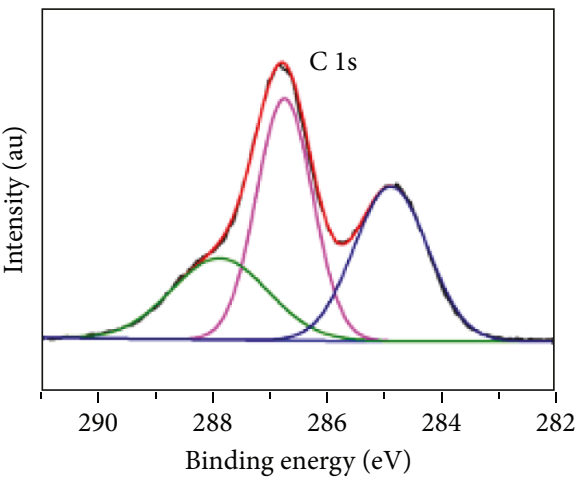

(e)

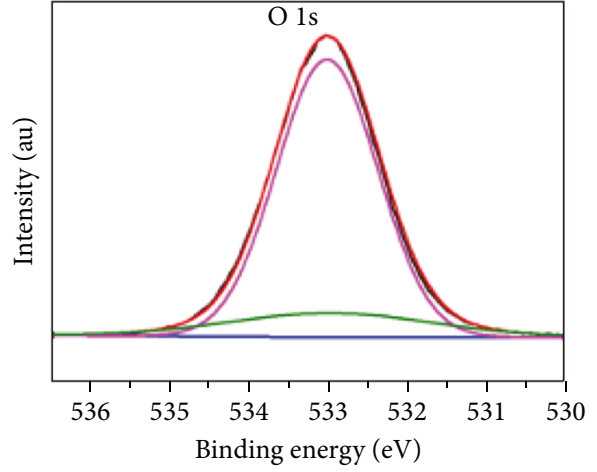

(b)

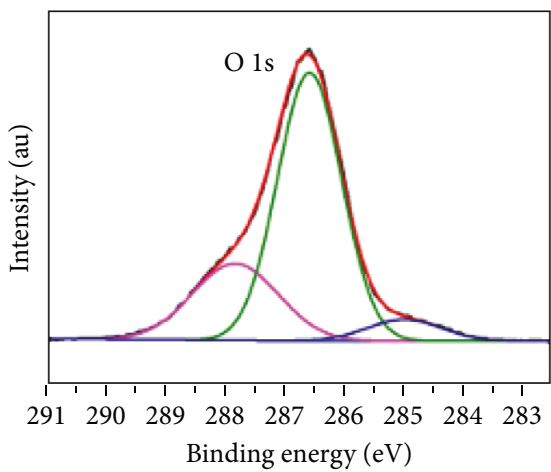

(d)

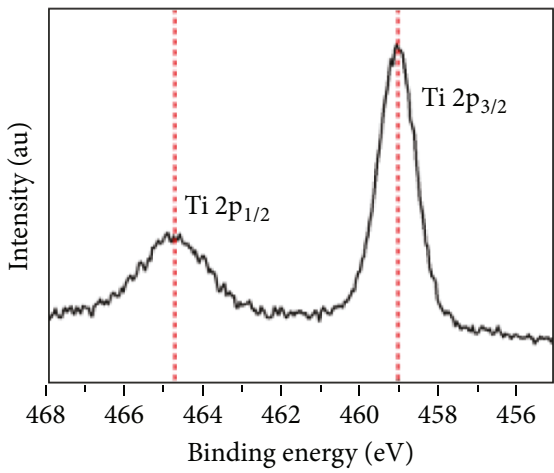

(f)

FIGURE 4: (a) A full-scan XPS spectra of CNF and CNFT, high-resolution XPS spectra of (b, c) O $1 \mathrm{~s}$ of CNF and CNFT, (d, e) C $1 \mathrm{~s}$ of CNF and CNFT, and (f) Ti $2 p$ of CNFT.

exhibited excellent crystallinity for the high and narrow peaks in the XRD patterns.

Figure 4 shows the XPS spectra of the CNF and CNFT2. The wide scan spectra of the CNF exhibited two major peaks with binding energy at $286.1 \mathrm{eV}$ and $532.1 \mathrm{eV}$ corresponding to the $\mathrm{C} 1 \mathrm{~s}$ and $\mathrm{O} 1 \mathrm{~s}$ of $\mathrm{CNF}$, respectively. However, in CNFT2, an additional peak was observed at a binding energy of $458.1 \mathrm{eV}$ corresponding to the $\mathrm{Ti} 2 \mathrm{p}$ of $\mathrm{TiO}_{2}$ [40]. The high-resolution XPS spectra were examined in order to research the structure further. As shown in Figure 4(b), two peaks were observed at $533 \mathrm{eV}$ in the high-resolution spectra of O 1s in CNF. Another peak was observed at $530.3 \mathrm{eV}$ in the high-resolution spectra of $\mathrm{O} 1 \mathrm{~s}$ in CNFT2 except the two peaks in the high-resolution spectra of $\mathrm{O} 1 \mathrm{~s}$ in the CNF at the same location. The additional peak could be attributed to $\mathrm{O}-\mathrm{Ti}^{4+}$. This further confirmed the presence of $\mathrm{TiO}_{2}$ in CNFT2. In both of the samples, three peaks were observed for $\mathrm{C}$ 1s in Figures 4(d) and 4(e) at the same location. The differences in the same peak on intensity at $285 \mathrm{eV}$ were attributed to $\mathrm{C}-\mathrm{C}$; the results may be some degradation during hydrothermal treatment. Furthermore, the binding energies of $\mathrm{Ti} 2 \mathrm{p}_{3 / 2}$ and $\mathrm{Ti} 2 \mathrm{p}_{1 / 2}$ appear at 459.02 and $464.92 \mathrm{eV}$, respectively (Figure 4(f)), which can be well attributed to the $\mathrm{Ti}^{4+}$ in bulk $\mathrm{TiO}_{2}$ [41]. According to the above results, it was obvious that $\mathrm{TiO}_{2}$ would have to be produced in CNFT successfully.

Figure 5 shows the different phase thermogravimetric and differential thermal analysis (TG-DTA) curves of CNF and CNFT2. In this study, the process of sample pyrolysis was divided into three stages, corresponding to drying (stage 1) 


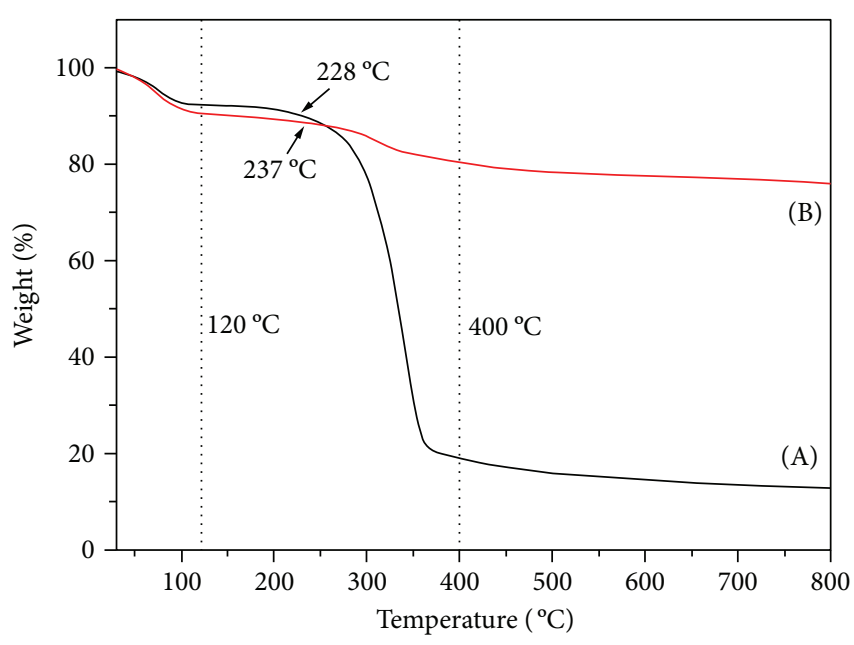

(a). $\mathrm{CNF}$

(b). CNFT

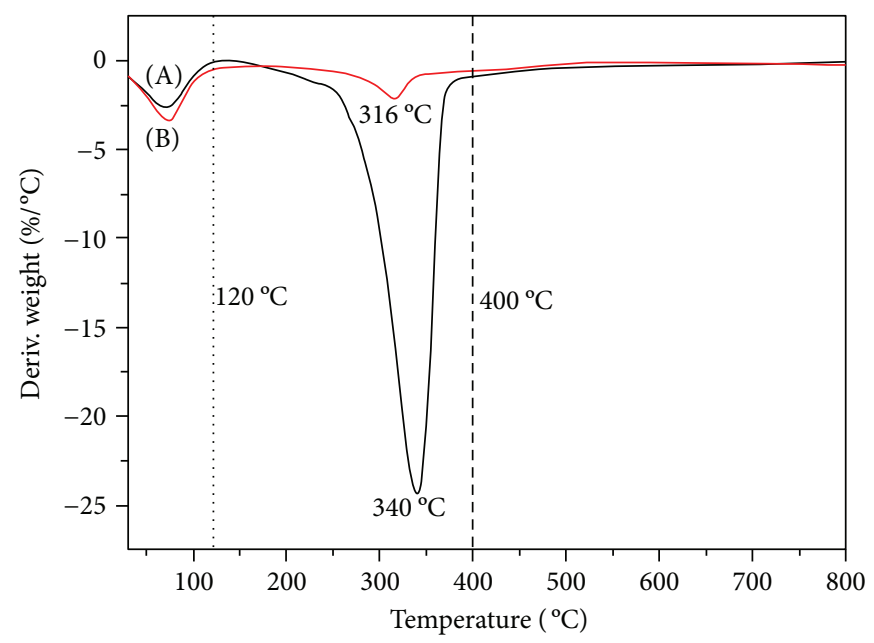

(a). $\mathrm{CNF}$

(b). CNFT

(a)

(b)

Figure 5: TGA and DTG thermograms of (a) CNF and (b) CNFT2 under nitrogen atmosphere.

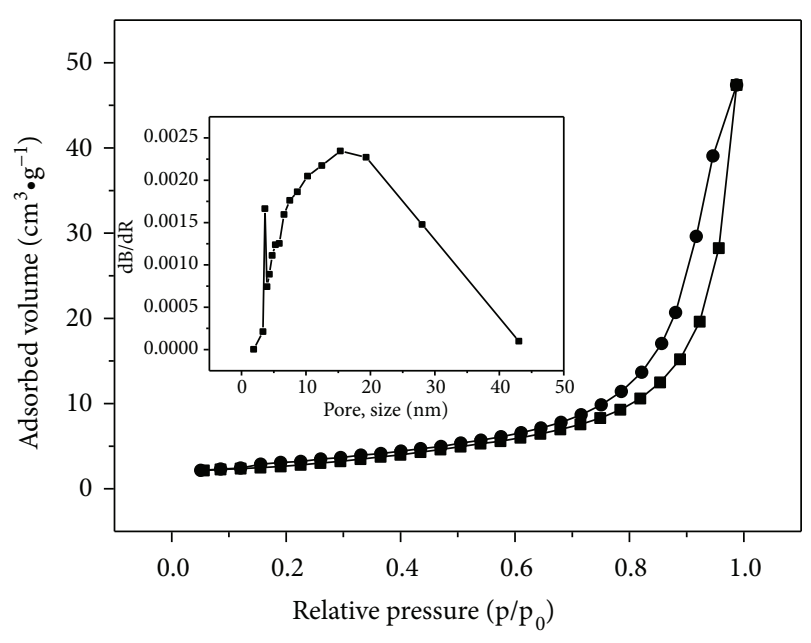

(a)

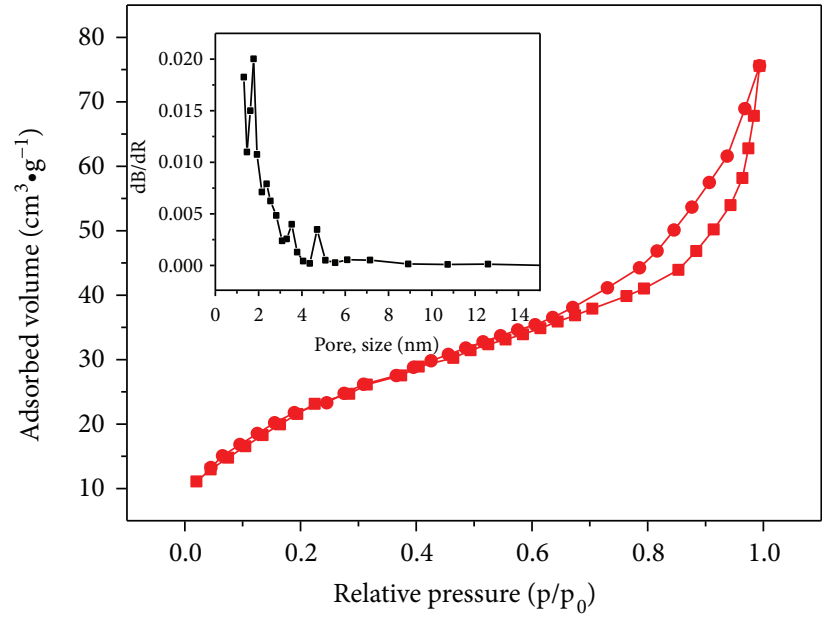

(b)

FIGURE 6: $\mathrm{N}_{2}$ adsorption/desorption isotherms of (a) CNF and (b) CNFT2 aerogels and corresponding pore size distribution derived from the adsorption branch [43].

TABLE 1: Texture properties of CNF and CNFT2.

\begin{tabular}{lccc}
\hline Sample & $\begin{array}{c}S_{\mathrm{BET}} \\
\left(\mathrm{m}^{2} \cdot \mathrm{g}^{-1}\right)\end{array}$ & $\begin{array}{c}V_{\text {total }} \\
\left(\mathrm{cm}^{3} \cdot \mathrm{g}^{-1}\right)\end{array}$ & $\begin{array}{c}\text { Average pore diameter } \\
(\mathrm{nm})\end{array}$ \\
\hline CNF & 10.1 & 0.073 & 29.3 \\
CNFT2 & 85.6 & 1.169 & 5.5 \\
\hline
\end{tabular}

and pyrolysis (stage 2 and 3 ). In the first stage, a small mass $(7 \%-9 \%)$ loss was observed in the temperature range of $25-120^{\circ} \mathrm{C}$, which due to the evaporation of water molecules in the materials or low molecular weight compounds for temperature increased. In the second stage $\left(120-400^{\circ} \mathrm{C}\right)$, some flat lines at the TG/DTG curves were displayed, and the beginning of weight loss of the main degradation step was observed at $228^{\circ} \mathrm{C}$ and $237^{\circ} \mathrm{C}$, corresponding to the curves of $\mathrm{CNF}$ and
CNFT2, respectively. Then, values (TG curve) of the samples decreased sharply with time. Importantly, the different components of CNF and CNFT2 caused great differences on the DTG curves. The rate of degradation reached its peak at $340^{\circ} \mathrm{C}$ and $316^{\circ} \mathrm{C}$ for $\mathrm{CNF}$ and $\mathrm{CNFT} 2$, respectively. The reason for this phenomenon may be attributed to the deposition of the $\mathrm{TiO}_{2}$ particles on the wood surface. Combining strongly with $\mathrm{TiO}_{2}$ particles via electrostatic attraction and hydrogen bond, the pyrolysis rate of CNFT2 decreased nearly tenfold as much as that of CNF, which effectively enhanced the thermal stability of CNFT2 [42]. In the third stage, the TG curves of CNF and CNFT2 were relatively flat. This result indicated that all the samples were degraded completely.

The $\mathrm{N}_{2}$ adsorption/desorption isotherms of $\mathrm{CNF}$ and CNFT2 aerogels displayed a direct hysteresis loop, shown 


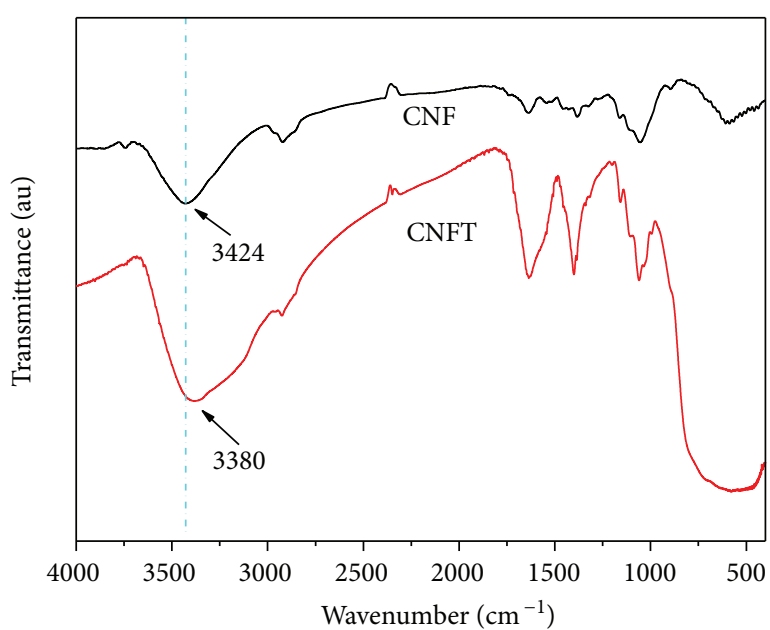

(a)

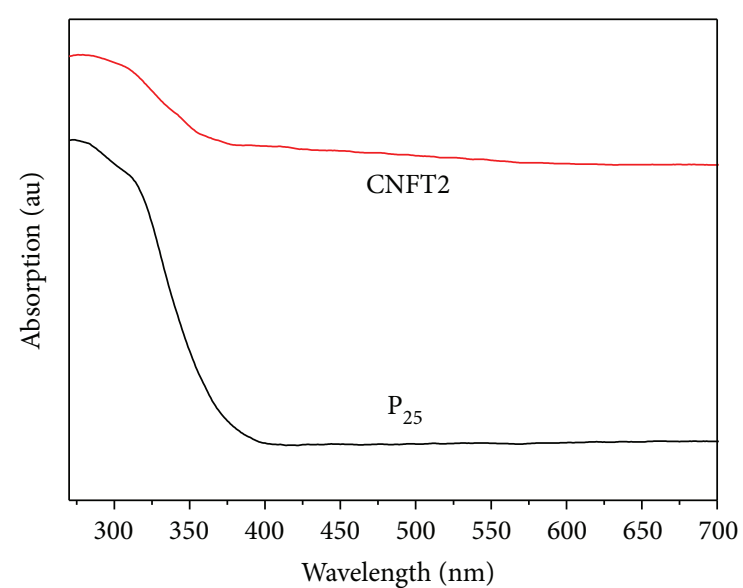

(b)

Figure 7: (a) FT-IR spectra of the CNF and CNFT2. (b) UV-Vis spectra of P25 and CNFT2.

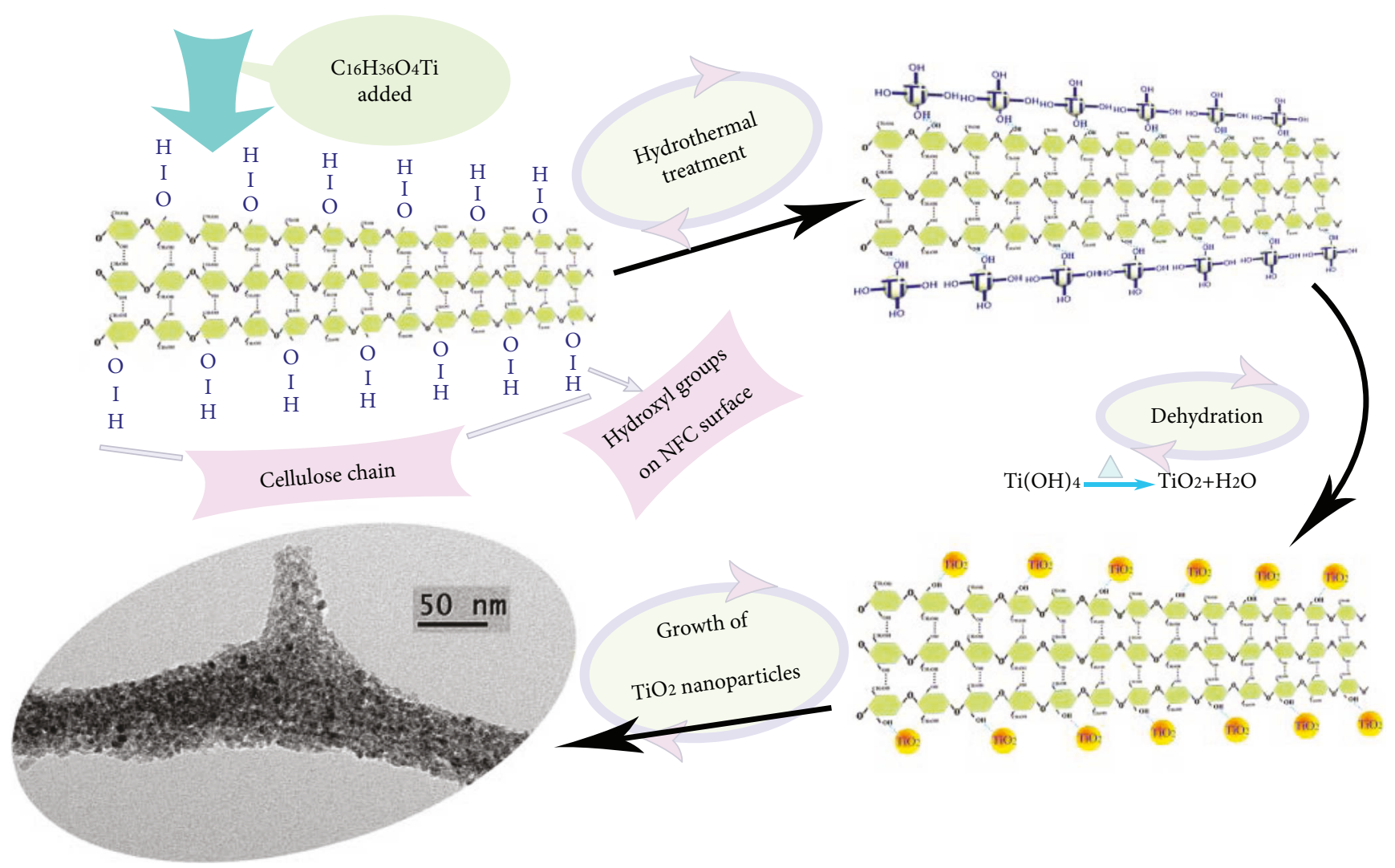

Figure 8: Mechanism for the generation of the $\mathrm{TiO}_{2}$ nanoparticles on the surface of CNF.

in Figure 6, belonging to type IV (according to IUPAC classification), which were consistent with the results researched by Fan et al.'s group [43]. The results reflected the presence of a mesoporous structure in the samples. The corresponding pore size distribution of the samples was calculated by the $\mathrm{BJH}$ method and was illustrated in the inset of Figure 6. The CNFT2 showed a smaller pore diameter (dominated in about $2 \mathrm{~nm}$ ) than did CNF (dominated about $15 \mathrm{~nm}$ ), but it also exhibited a sharper and more concentrated peak. The porosity detailed data of CNF and CNFT2 is summarized in Table 1 . It could be seen that the specific surface of CNFT2 was 8 times higher than that of CNF, and the pore volume of CNFT2 was 2 orders of magnitude above that of CNF.

Figure 7(a) shows the FT-IR spectra of CNF and CNFT2. The dominant peak of CNF in the region around $3424 \mathrm{~cm}^{-1}$ was attributed to stretching vibrations of hydroxyl groups of cellulose. However, in CNFT2, the peak at $3380 \mathrm{~cm}^{-1}$ 


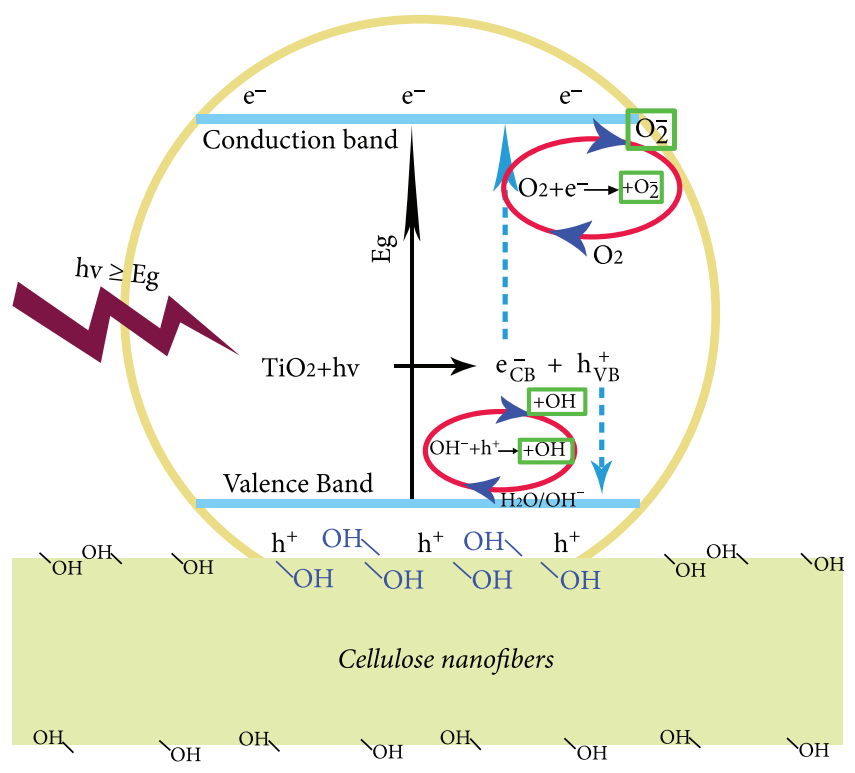

FIGURE 9: Schematic illustrating the strategies to develop $\bullet \mathrm{O}_{2}$ and $\bullet \mathrm{OH}$.

was shifted to lower wavenumbers, indicating a strong interaction between the hydroxyl groups of cellulose and $\mathrm{TiO}_{2}$ nanoparticles through a hydrogen bond [44]. This strong interaction led to $\mathrm{TiO}_{2}$ nanoparticles adhering to the surface of CNF. Figure 7(b) shows the UV-Vis spectra of P25 and CNFT2. An obvious red shift could be observed on the cut-off wavelength from 385 to $411 \mathrm{~nm}$ of CNFT2, compared to P25. The result indicated the band gap of CNFT2 triggered by hydrogen bonds between P25 and CNF. The band gap energy $\left(E_{\mathrm{g}}\right)$ was obtained according to [45]

$$
E_{\mathrm{g}}=h \bullet \frac{C}{\lambda}
$$

where $h=$ plank constant $=6.626 \times 10^{-34}$ joules $/ \mathrm{sec}, C=$ speed of light $=3.0 \times 10^{8}$ meters $/ \mathrm{sec}, \lambda=$ cut-off wavelength at $1 \mathrm{eV}$ $=1.6 \times 10^{-19}$ joules. For CNFT2, the band gap energy about $3.023 \mathrm{eV}$ was lower than that of the standard commercial P25 $(3.227 \mathrm{eV})$, indicating that CNFT2 was able to absorb photons not only from UV irradiation but also from visible light. The extended light absorption range made the CNFT2 composites good candidates for visible light photocatalysis.

The interaction of $\mathrm{TiO}_{2}$ nanoparticles and the surface of $\mathrm{CNF}$ are shown in Figure 8. As we all know, there were plenty of hydroxyl groups present on the surface of CNF [46]. When $\mathrm{C}_{16} \mathrm{H}_{36} \mathrm{O}_{4}$ Ti was added in the solution, $\mathrm{Ti}(\mathrm{OH})_{4}$ could generate due to the hydrolysis of $\mathrm{C}_{16} \mathrm{H}_{36} \mathrm{O}_{4} \mathrm{Ti}$. $\mathrm{Ti}(\mathrm{OH})_{4}$ molecules could be interacted with the surface of CNF by the hydrogen bond between the hydroxyl groups on the surface of CNF and the hydroxyl groups of $\mathrm{Ti}(\mathrm{OH})_{4}$, which was consistent with the result of the FT-IR. On dehydration, $\mathrm{TiO}_{2}$ could be generated through losing $\mathrm{H}_{2} \mathrm{O}$ among the hydroxyl groups in $\mathrm{Ti}(\mathrm{OH})_{4} \cdot \mathrm{TiO}_{2}$ and $\mathrm{CNF}$ would be combined together due to the existence of hydrogen bonds between the hydroxyl groups on the surface of $\mathrm{TiO}_{2}$ and CNF. This further helped in the generation of the $\mathrm{TiO}_{2}$ nuclei on the surface of the $\mathrm{CNF}$. As the reaction proceeded, the nuclei would grow and form $\mathrm{TiO}_{2}$ nanoparticles on the surface of the CNF.

Figure 9 illustrates the detailed performance of the photodegradation procedure of CNFT. The basic species responsible for the photodegradation of any pollutant were hydroxyl radicals and anion radical superoxide. Electron $\left(\mathrm{e}_{\mathrm{CB}}{ }^{-}\right)$and hole $\left(\mathrm{h}_{\mathrm{VB}}{ }^{+}\right)$pairs with excited high-energy states occurred when wide band gap semiconductors were irradiated higher than their band gap energy [47]. Firstly, the excitation of electrons occurred from the valance band to the conduction band by the photons emanated from the UV source, and then the holes generated due to the excitation process acted as a decomposing agent or combined with the surface hydroxyl species on the $\mathrm{TiO}_{2}$ to form the hydroxyl radical. $\mathrm{h}_{\mathrm{VB}}{ }^{+}$and $\mathrm{e}_{\mathrm{CB}}{ }^{-}$were powerfully oxidizing. $\mathrm{h}_{\mathrm{VB}}{ }^{+}$reacted with $\mathrm{OH}^{-}$contained in water resulting in their oxidation producing $\bullet \mathrm{OH}$. $\mathrm{e}_{\mathrm{CB}}{ }^{-}$could be reacted with $\mathrm{O}_{2}$ forming an anion radical superoxide. The organic compounds were oxidized to $\mathrm{CO}_{2}$ and $\mathrm{H}_{2} \mathrm{O}$. However, it should be noted that the continuous reactions during photocatalytic degradation were attributed to the presence of dissolved oxygen and water molecules [48]. Cellulose played an important role in the CNFT composite when the procedure of photodegradation was conducted. It would provide innumerable hydroxyl groups on the surface, which could capture the holes and stop the electron-hole from recombining. The electron and hole pairs could be separated to conduce and continue the reaction of oxidation.

Figure 10 shows the performance of photodegradation of the CNFT composite. $\mathrm{TiO}_{2}$ had great potential application in environmental protection. In this study, the potential application of CNFT was investigated to degrade dye under UV light and sunlight. Methylene blue (MB) was chosen as a model dye. The time-dependent UV-Vis optical absorption of MB and its degradation by CNFT2 under UV light is shown in Figure 10(a). The inset shows the colorless MB aqueous solution under UV light as a function of time. MB solutions containing CNFT2 were degraded almost absolutely within $20 \mathrm{~min}$. The colors faded gradually in solution for dye degradation with time. After 5 minutes, the color turned to be shallow and became colorless after about 20 minutes. It indicated that $\mathrm{MB}$ was degraded almost completely for about 20 minutes. The results were consistent with the relevant UV-Vis spectra as a function of time in the inset of Figure 10(a). In addition, the as-prepared aerogels (CNF and CNFTs) immersed in $\mathrm{MB}$ solution were compared in darkness (Figure 10(b)). It was observed that the decrease in absorption could be reasonable due to the adsorption on the surface. The decrease in absorption could result from adsorption on the surfaces of aerogels. However, the adsorption capacity of CNFT was higher than that of CNF because of the higher specific surface area. The results could conclude that CNFT possessed a special nanostructure and enhanced the dye adsorption. In contrast, UV light irradiation for $30 \mathrm{~min}$ is shown in Figure 10(c). A self-decomposition phenomenon of $14.8 \%$ could be seen in the $\mathrm{MB}$ solution without anything. About $27.5 \%$ of $\mathrm{MB}$ had been degraded in the case of CNF. However, the as-prepared CNFT exhibited much better photocatalytic activity. Moreover, the property of photodegradation of the composite 

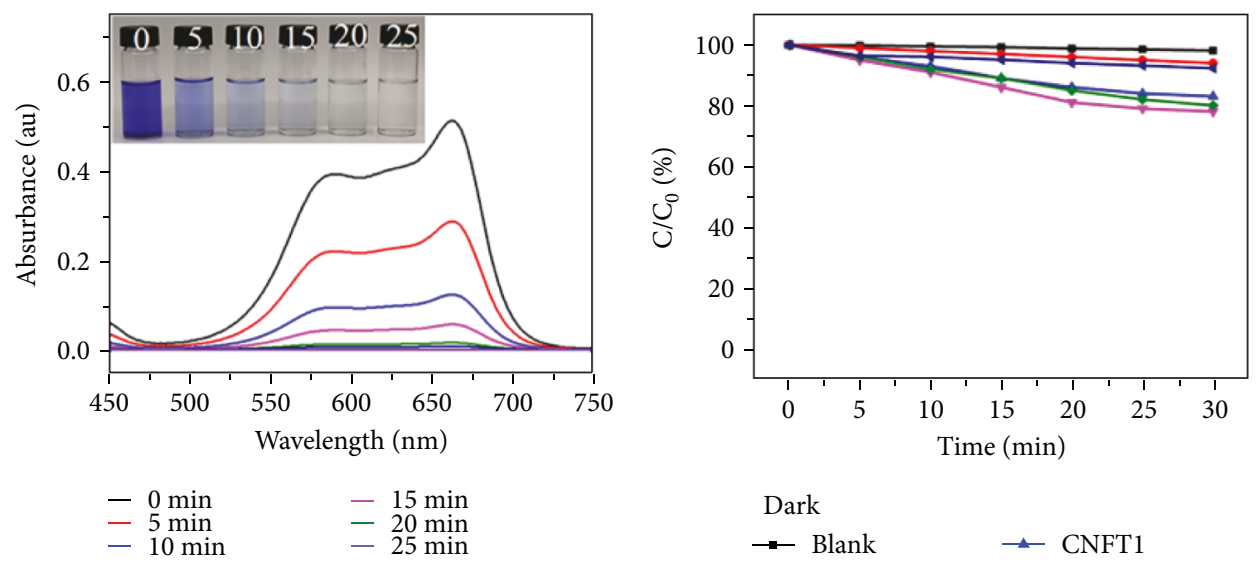

Dark

$\rightarrow \mathrm{CNF}$

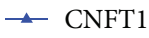

$\longleftarrow \mathrm{TiO}_{2}$

$\checkmark$ CNFT2

(a)

(b)

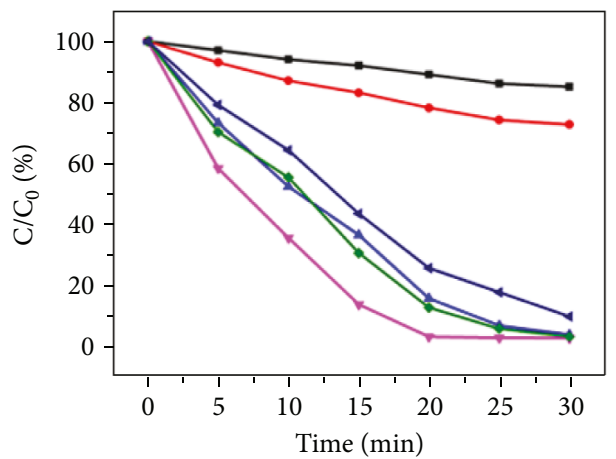

UV

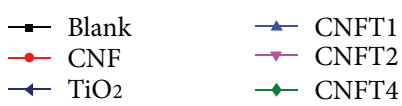

(c)

FIgUre 10: (a) Time-dependent UV-visible spectra of MB and its degradation by CNFT2 under UV irradiation; the colors in different times were shown in the inset. (b) Quantitative study of photocatalytic degradation of $\mathrm{MB}$ by blank, $\mathrm{CNF}, \mathrm{TiO}_{2}$, and as-prepared $\mathrm{CNFT}$ as a function in darkness and (c) under UV irradiation.

TABLE 2: Summary of results for degradation of $\mathrm{MB}$ employed by modified $\mathrm{TiO}_{2}$ under UV light.

\begin{tabular}{|c|c|c|c|c|c|}
\hline Photocatalyst & Modified type & Power $(\mathrm{W})$ & Photodegradation rate (\%) & Time (min) & Ref. \\
\hline $\mathrm{TiO}_{2}$ & $\mathrm{Pd}$ & 300 & 98 & 60 & {$[49]$} \\
\hline $\mathrm{TiO}_{2}$ & $\mathrm{Ag}_{2} \mathrm{O}$ & 160 & 97 & 360 & {$[50]$} \\
\hline $\mathrm{TiO}_{2}$ & $\mathrm{Fe}_{2} \mathrm{O}_{3}$ & 400 & 81 & 100 & {$[51]$} \\
\hline $\mathrm{TiO}_{2}$ & $\mathrm{Ag}-\mathrm{Ag}_{2} \mathrm{O}$ & 112 & 99.62 & 200 & {$[52]$} \\
\hline $\mathrm{TiO}_{2}$ & Carbon nanotubes & 125 & 76 & 200 & [53] \\
\hline $\mathrm{TiO}_{2}$ & Cellulose & 1000 & 98.1 & 20 & This work \\
\hline $\mathrm{TiO}_{2}$ & Cellulose & 500 & 96.7 & 45 & This work \\
\hline $\mathrm{TiO}_{2}$ & Cellulose & 200 & 89.6 & 200 & This work \\
\hline
\end{tabular}

CNFT2 was better than the others. Contributing to the interaction of cellulose and $\mathrm{TiO}_{2}, 98.1 \% \mathrm{MB}$ dye was degraded by the as-prepared CNFT2 only for 20 minutes, and 97\% and $97.7 \%$ MB dye were degraded by CNFT1 and CNFT4 for 30 minutes. Furthermore, $92.3 \% \mathrm{MB}$ dye was degraded by the as-prepared $\mathrm{TiO}_{2}$ for 30 minutes. This observation would suggest that CNFT could enhance the functionality through a synergistic interaction between the $\mathrm{CNF}$ and $\mathrm{TiO}_{2}$ nanoparticles due to the interactive structure in materials. On the other hand, in order to increase the photocatalytic activity, many researches had been conducted in the previous investigation. The summary of results for MB degradation 


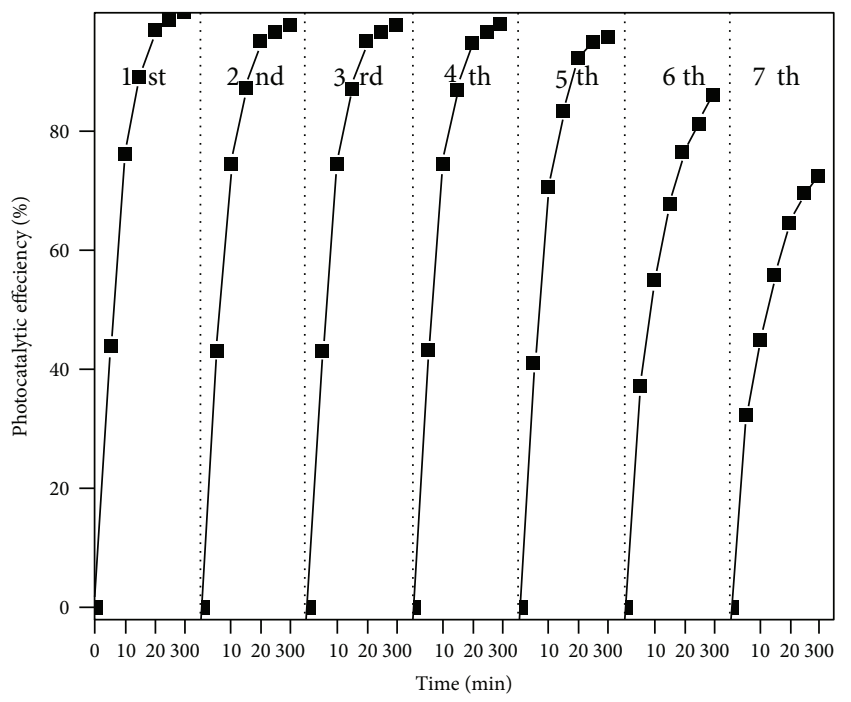

(a)

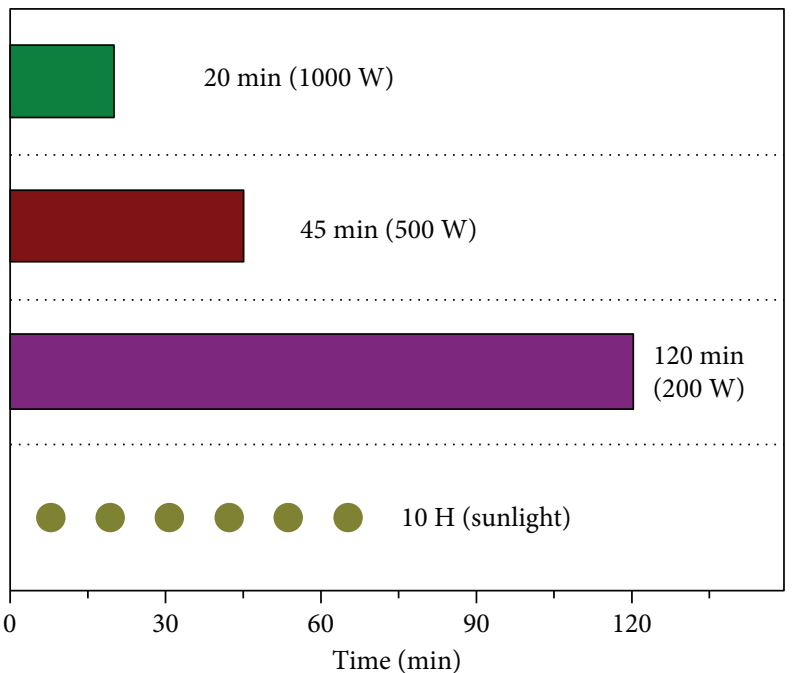

(b)

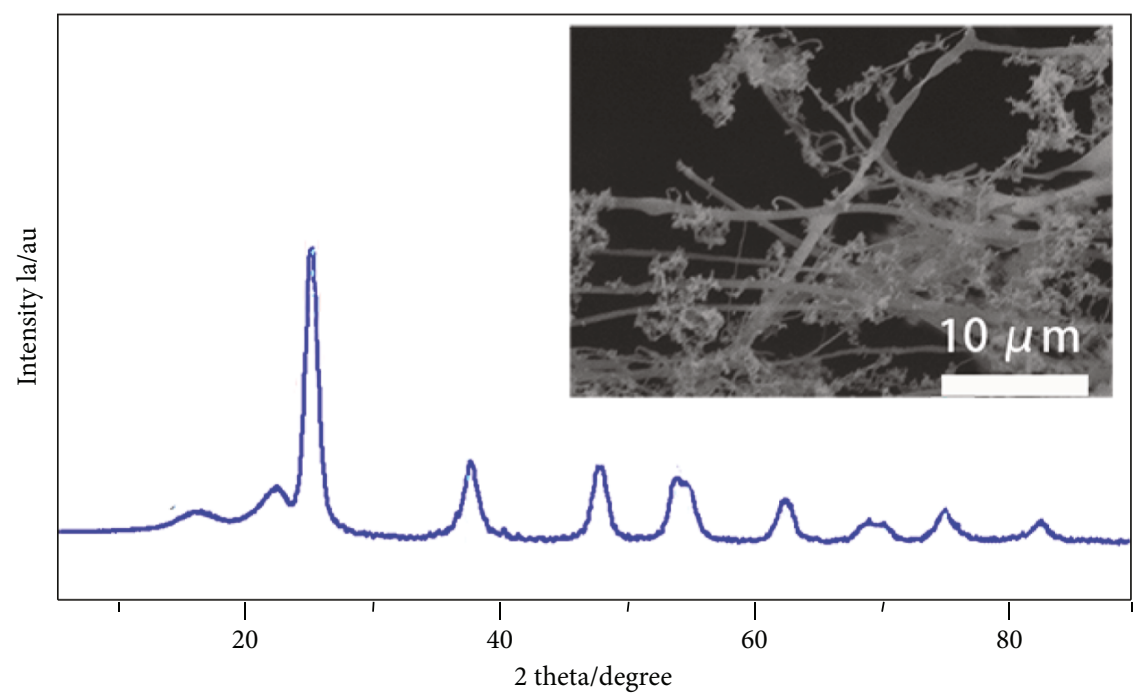

(c)

FIGURE 11: (a) Seven cycles of the photodegradation of MB using CNFT2 as a photocatalyst under UV irradiation for 30 min. (b) Degradation of MB with different light intensities of UV irradiation and sunlight. (c) XRD pattern and SEM image of the CNFT2 after the 7th recycle.

employed by modified $\mathrm{TiO}_{2}$ with different components in different conditions is shown in Table 2.

The stability and reusability of the CNFT2, as a typical representative, were investigated and exhibited by performing several UV irradiation cycles. In each cycle, simulated UV light was irradiated for $30 \mathrm{~min}$ at room temperature in Figure 11(a). Obviously, CNFT2 could keep a superior photocatalytic performance with the degradation beyond $98 \%$ after 4 photocatalytic cycles. After the 7 th photocatalytic recycle finished, the photodegradation of CNFT2 decreased to about $74 \%$, which was due to about $19 \%$ mass loss of CNFT2 during the collection process. Hence, the decreased photocatalytic activity of CNFT2 after multiple cycles was contributed to the mass loss of CNFT2. Compared with other research results, CNFT2 showed an excellent stability and reusability. Furthermore, MB solution with CNFT2 was exposed to UV irradiation produced by a tunable pressure $\mathrm{Hg}$ lamp, at
$1000 \mathrm{~W}, 500 \mathrm{~W}$, and $200 \mathrm{~W}$, and sunlight outdoor for $1 \mathrm{~h}$ in the noon every sunny day. Figure 11(b) shows that MB could be well degraded with different light intensities of UV irradiation and sunlight. The results indicated that CNFT2 exhibited excellent properties of photodegradation not only under UV irradiation but also under sunlight which could cause the MB to photodegrade. As shown in Figure 11(c), the results of the XRD pattern and SEM image of CNFT2 after the 7th recycle were not obviously different from that of CNFT2 before being used for photocatalytic reaction, which illustrated the stability of CNFT2.

\section{Conclusions}

Cellulose nanofiber/ $/ \mathrm{TiO}_{2}$ aerogels were successfully fabricated, which the obtained $\mathrm{TiO}_{2}$ nanoparticles adhered to the surface of the cellulose nanofiber as scaffold through a 
hydrogen bond. The composites of CNFT were good enough for photocatalytic applications and even much better than CNF and individual $\mathrm{TiO}_{2}$ nanoparticles. This observation would suggest that CNFT could enhance the functionality through a synergistic interaction between the CNF and $\mathrm{TiO}_{2}$ nanoparticles due to the interactive structure in materials. The performance might lead to the fabrication of new materials that could be used for multiple activities ideal for water treatment applications as $\mathrm{TiO}_{2}$ was nontoxic and efficient in preventing infections. Furthermore, the composite aerogel could be regarded as a green and portable photocatalyst because it was promising for wastewater treatment application as a result of its good high photocatalytic activity and stability under UV light irradiation. The one-step in situ method could be generalized to the incorporation of many more metal/metal oxide nanostructures in cellulose products.

\section{Data Availability}

The data used to support the findings of this study are available from the corresponding author upon request.

\section{Conflicts of Interest}

The authors declare that there are no conflicts of interest regarding the publication of this paper.

\section{Acknowledgments}

The work was financially supported by a project supported by the Natural Science Foundation of Hunan Province (Grant no. 2016JJ3185).

\section{References}

[1] R. Fagan, D. Mccormack, S. Hinder, and S. Pillai, "Photocatalytic properties of $\mathrm{g}-\mathrm{C}_{3} \mathrm{~N}_{4}-\mathrm{TiO}_{2}$ heterojunctions under UV and visible light conditions," Materials, vol. 9, no. 4, pp. 286-291, 2016.

[2] Q. Wang, M. Zhang, C. Chen, W. Ma, and J. Zhao, "Photocatalytic aerobic oxidation of alcohols on $\mathrm{TiO}_{2}$ : the acceleration effect of a Brønsted acid," Angewandte Chemie International Edition, vol. 49, no. 43, pp. 7976-7979, 2010.

[3] Z. Liu, X. Zhang, S. Nishimoto et al., "Highly ordered $\mathrm{TiO} 2$ nanotube arrays with controllable length for photoelectrocatalytic degradation of phenol," The Journal of Physical Chemistry C, vol. 112, no. 1, pp. 253-259, 2007.

[4] A. T. Kuvarega, R. W. M. Krause, and B. B. Mamba, "Nitrogen/ palladium-codoped $\mathrm{TiO} 2$ for efficient visible light photocatalytic dye degradation," Journal of Physical Chemistry C, vol. 115, no. 45, pp. 22110-22120, 2011.

[5] Q. Qu, H. Geng, R. Peng et al., "Chemically binding carboxylic acids onto $\mathrm{TiO}_{2}$ nanoparticles with adjustable coverage by solvothermal strategy," Langmuir, vol. 26, no. 12, pp. 9539-9546, 2010.

[6] K. Lv, J. Yu, K. Deng, X. Li, and M. Li, "Effect of phase structures on the formation rate of hydroxyl radicals on the surface of $\mathrm{TiO}_{2}$," Journal of Physics and Chemistry of Solids, vol. 71, no. 4, pp. 519-522, 2010.
[7] L. Karimi, M. E. Yazdanshenas, R. Khajavi, A. Rashidi, and M. Mirjalili, "Using graphene/ $/ \mathrm{TiO}_{2}$ nanocomposite as a new route for preparation of electroconductive, self-cleaning, antibacterial and antifungal cotton fabric without toxicity," Cellulose, vol. 21, no. 5, pp. 3813-3827, 2014.

[8] J. Hofkens and M. B. J. Roeffaers, "Electrochemistry: photocatalysts in close-up," Nature, vol. 530, no. 7588, pp. 36-37, 2016.

[9] H. Li, H. Liu, A. Fu et al., "Synthesis and characterization of $\mathrm{N}$-doped porous $\mathrm{TiO}_{2}$ hollow spheres and their photocatalytic and optical properties," Materials, vol. 9, no. 10, pp. 849-854, 2016.

[10] H. Huang, H. Huang, L. Zhang, H. Peng, X. Ye, and D. Y. C. Leung, "Enhanced degradation of gaseous benzene under vacuum ultraviolet (VUV) irradiation over $\mathrm{TiO} 2$ modified by transition metals," Chemical Engineering Journal, vol. 259, pp. 534-541, 2015.

[11] J. Ren, S. Wang, C. Gao, X. Chen, W. Li, and F. Peng, “ $\mathrm{TiO}_{2}$ -containing PVA/xylan composite films with enhanced mechanical properties, high hydrophobicity and UV shielding performance," Cellulose, vol. 22, no. 1, pp. 593-602, 2015.

[12] C. Moreno-Castilla and F. J. Maldonado-Hódar, "Carbon aerogels for catalysis applications: an overview," Carbon, vol. 43, no. 3, pp. 455-465, 2005.

[13] J. Biener, M. Stadermann, M. Suss et al., "Advanced carbon aerogels for energy applications," Energy \& Environmental Science, vol. 4, no. 3, pp. 656-667, 2011.

[14] W. Chen, Q. Li, Y. Wang et al., "Comparative study of aerogels obtained from differently prepared nanocellulose fibers," ChemSusChem, vol. 7, no. 1, pp. 154-161, 2014.

[15] J. Zou, J. Liu, A. S. Karakoti et al., "Ultralight multiwalled carbon nanotube aerogel," ACS Nano, vol. 4, no. 12, pp. 7293-7302, 2010.

[16] C. Wu, X. Huang, X. Wu, R. Qian, and P. Jiang, "Mechanically flexible and multifunctional polymer-based graphene foams for elastic conductors and oil-water separators," Advanced Materials, vol. 25, no. 39, pp. 5658-5662, 2013.

[17] H.-W. Liang, Q.-F. Guan, L.-F. Chen, Z. Zhu, W.-J. Zhang, and S.-H. Yu, "Macroscopic-scale template synthesis of robust carbonaceous nanofiber hydrogels and aerogels and their applications," Angewandte Chemie, vol. 124, no. 21, pp. 5191-5195, 2012.

[18] Z. Xu, Y. Zhang, P. Li, and C. Gao, "Strong, conductive, lightweight, neat graphene aerogel fibers with aligned pores," ACS Nano, vol. 6, no. 8, pp. 7103-7113, 2012.

[19] S. Yin, Z. Niu, and X. Chen, "Assembly of graphene sheets into 3D macroscopic structures," Small, vol. 8, no. 16, pp. 2458-2463, 2012.

[20] E. Abraham, B. Deepa, L. A. Pothan et al., "Physicomechanical properties of nanocomposites based on cellulose nanofibre and natural rubber latex," Cellulose, vol. 20, no. 1, pp. 417-427, 2013.

[21] N. C. T. Martins, C. S. R. Freire, R. J. B. Pinto et al., "Electrostatic assembly of Ag nanoparticles onto nanofibrillated cellulose for antibacterial paper products," Cellulose, vol. 19, no. 4, pp. 1425-1436, 2012.

[22] W. Chen, H. Yu, Q. Li, Y. Liu, and J. Li, "Ultralight and highly flexible aerogels with long cellulose I nanofibers," Soft Matter, vol. 7, no. 21, pp. 10360-10368, 2011.

[23] M. Pääkkö, J. Vapaavuori, R. Silvennoinen et al., "Long and entangled native cellulose I nanofibers allow flexible aerogels 
and hierarchically porous templates for functionalities," Soft Matter, vol. 4, no. 12, pp. 2492-2499, 2008.

[24] A. C. Borges, C. Eyholzer, F. Duc et al., "Nanofibrillated cellulose composite hydrogel for the replacement of the nucleus pulposus," Acta Biomaterialia, vol. 7, no. 9, pp. 3412-3421, 2011.

[25] R. Xiong, C. Lu, Y. Wang, Z. Zhou, and X. Zhang, "Nanofibrillated cellulose as the support and reductant for the facile synthesis of $\mathrm{Fe}_{3} \mathrm{O}_{4} / \mathrm{Ag}$ nanocomposites with catalytic and antibacterial activity," Journal of Materials Chemistry A, vol. 1, no. 47, pp. 14910-14918, 2013.

[26] M. Timusk, M. Järvekülg, A. Salundi et al., "Optical properties of high-performance liquid crystal-xerogel microcomposite electro-optical film," Journal of Materials Research, vol. 27, no. 9, pp. 1257-1264, 2012.

[27] B. Deepa, E. Abraham, L. Pothan, N. Cordeiro, M. Faria, and S. Thomas, "Biodegradable nanocomposite films based on sodium alginate and cellulose nanofibrils," Materials, vol. 9, no. 1, pp. 50-57, 2016.

[28] T. Wang and L. T. Drzal, "Cellulose-nanofiber-reinforced poly(lactic acid) composites prepared by a water-based approach," ACS Applied Materials \& Interfaces, vol. 4, no. 10, pp. 5079-5085, 2012.

[29] S. Belbekhouche, J. Bras, G. Siqueira et al., "Water sorption behavior and gas barrier properties of cellulose whiskers and microfibrils films," Carbohydrate Polymers, vol. 83, no. 4, pp. 1740-1748, 2011.

[30] S. Tidjarat, W. Winotapun, P. Opanasopit, T. Ngawhirunpat, and T. Rojanarata, "Uniaxially aligned electrospun cellulose acetate nanofibers for thin layer chromatographic screening of hydroquinone and retinoic acid adulterated in cosmetics," Journal of Chromatography A, vol. 1367, pp. 141-147, 2014.

[31] T. W. Kim, Y. Yang, F. Li, and W. L. Kwan, "Electrical memory devices based on inorganic/organic nanocomposites," NPG Asia Materials, vol. 4, no. 6, p. e18, 2012.

[32] J. Yuan, Y. Xu, and A. H. E. Müller, "One-dimensional magnetic inorganic-organic hybrid nanomaterials," Chemical Society Reviews, vol. 40, no. 2, pp. 640-655, 2011.

[33] W.-Q. Fan, J. Feng, S.-Y. Song, Y.-Q. Lei, G.-L. Zheng, and H.-J. Zhang, "Synthesis and optical properties of europiumcomplex-doped inorganic/organic hybrid materials built from oxo-hydroxo organotin nano building blocks," Chemistry - A European Journal, vol. 16, no. 6, pp. 1903-1910, 2010.

[34] Q. Yao, Y. Xiong, H. Wang, C. Wang, and Q. Sun, "MnO2nanoflakes/cellulose nanofibre aerogel fabricated via ultrasonication for high-performance water desalination," Journal of Materials Chemistry A, vol. 5, no. 20, pp. 9580-9590, 2017.

[35] Y. Xiong, C. Wang, H. Wang et al., “A 3D titanate aerogel with cellulose as the adsorption-aggregator for highly efficient water purification," Journal of Materials Chemistry A, vol. 5, no. 12, pp. 5813-5819, 2017.

[36] Z. Cheng and J. Lin, "Layered organic-inorganic hybrid perovskites: structure, optical properties, film preparation, patterning and templating engineering," CrystEngComm, vol. 12, no. 10, 2010.

[37] S. Xiao, R. Gao, Y. Lu, J. Li, and Q. Sun, "Fabrication and characterization of nanofibrillated cellulose and its aerogels from natural pine needles," Carbohydrate Polymers, vol. 119, no. 119, pp. 202-209, 2015.

[38] R. Bezerra, M. Silva, A. Morais et al., "Phosphated cellulose as an efficient biomaterial for aqueous drug ranitidine removal," Materials, vol. 7, no. 12, pp. 7907-7924, 2014.
[39] N. Cai, X. Wang, D. Guo et al., “Titanium dioxide fibers prepared from two novel polytitanium precursors containing linear $\mathrm{Ti}-\mathrm{OH}-\mathrm{Ti}$ chains applied for photocatalytic degradation," Materials Letters, vol. 153, pp. 191-194, 2015.

[40] X. Liu, Y. Li, J. Yang et al., "Enhanced photocatalytic activity of CdS-decorated $\mathrm{TiO}_{2} /$ carbon core-shell microspheres derived from microcrystalline cellulose," Materials, vol. 9, no. 4, pp. 245-231, 2016.

[41] I. Chauhan and P. Mohanty, "In situ decoration of $\mathrm{TiO}_{2}$ nanoparticles on the surface of cellulose fibers and study of their photocatalytic and antibacterial activities," Cellulose, vol. 22, no. 1, pp. 507-519, 2015.

[42] J. Zeng, S. Liu, J. Cai, and L. Zhang, “TiO2 immobilized in cellulose matrix for photocatalytic degradation of phenol under weak UV light irradiation," The Journal of Physical Chemistry C, vol. 114, no. 17, pp. 7806-7811, 2010.

[43] B. Fan, S. Chen, Q. Yao, Q. Sun, and C. Jin, "Fabrication of cellulose nanofiber/AlOOH aerogel for flame retardant and thermal insulation," Materials, vol. 10, no. 3, p. 311, 2017.

[44] M. Poletto, H. Ornaghi Júnior, and A. Zattera, "Native cellulose: structure, characterization and thermal properties," Materials, vol. 7, no. 9, pp. 6105-6119, 2014.

[45] M. K. Debanath and S. Karmakar, "Study of blueshift of optical band gap in zinc oxide $(\mathrm{ZnO})$ nanoparticles prepared by lowtemperature wet chemical method," Materials Letters, vol. 111, no. 45, pp. 116-119, 2013.

[46] C. Aulin, J. Netrval, L. Wågberg, and T. Lindström, “Aerogels from nanofibrillated cellulose with tunable oleophobicity," Soft Matter, vol. 6, no. 14, pp. 3298-3305, 2010.

[47] X. Lang, W. Ma, C. Chen, H. Ji, and J. Zhao, "Selective aerobic oxidation mediated by $\mathrm{TiO} 2$ photocatalysis," Accounts of Chemical Research, vol. 47, no. 2, pp. 355-363, 2013.

[48] N. de la Cruz, V. Romero, R. F. Dantas et al., "o-Nitrobenzaldehyde actinometry in the presence of suspended $\mathrm{TiO}_{2}$ for photocatalytic reactors," Catalysis Today, vol. 209, no. 12, pp. 209-214, 2013.

[49] M. Y. Abdelaal and R. M. Mohamed, "Novel Pd/TiO nanocomposite prepared by modified sol-gel method for photocatalytic degradation of methylene blue dye under visible light irradiation," Journal of Alloys and Compounds, vol. 576, pp. 201-207, 2013.

[50] F. Chen, Z. Liu, Y. Liu, P. Fang, and Y. Dai, "Enhanced adsorption and photocatalytic degradation of high-concentration methylene blue on $\mathrm{Ag}_{2} \mathrm{O}$-modified $\mathrm{TiO}_{2}$-based nanosheet," Chemical Engineering; Journal, vol. 221, no. 2, pp. 283-291, 2013.

[51] A. Abbasi, D. Ghanbari, M. Salavati-Niasari, and M. Hamadanian, "Photo-degradation of methylene blue: photocatalyst and magnetic investigation of $\mathrm{Fe}_{2} \mathrm{O}_{3}-\mathrm{TiO}_{2}$ nanoparticles and nanocomposites," Journal of Materials Science: Materials in Electronics, vol. 27, no. 5, pp. 4800-4809, 2016.

[52] R. Kumar, R. El-Shishtawy, and M. Barakat, "Synthesis and characterization of ag- $\mathrm{Ag}_{2} \mathrm{O} / \mathrm{TiO}_{2} @$ polypyrrole heterojunction for enhanced photocatalytic degradation of methylene blue," Catalysts, vol. 6, no. 6, 2016.

[53] D. Zhao, X. Yang, C. Chen, and X. Wang, "Enhanced photocatalytic degradation of methylene blue on multiwalled carbon nanotubes- $\mathrm{TiO}_{2}$," Journal of Colloid and Interface Science, vol. 398, pp. 234-239, 2013. 


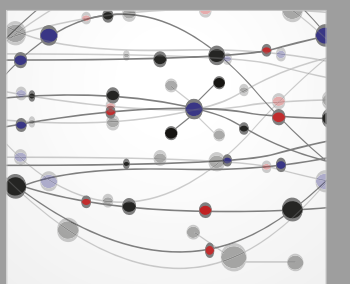

The Scientific World Journal
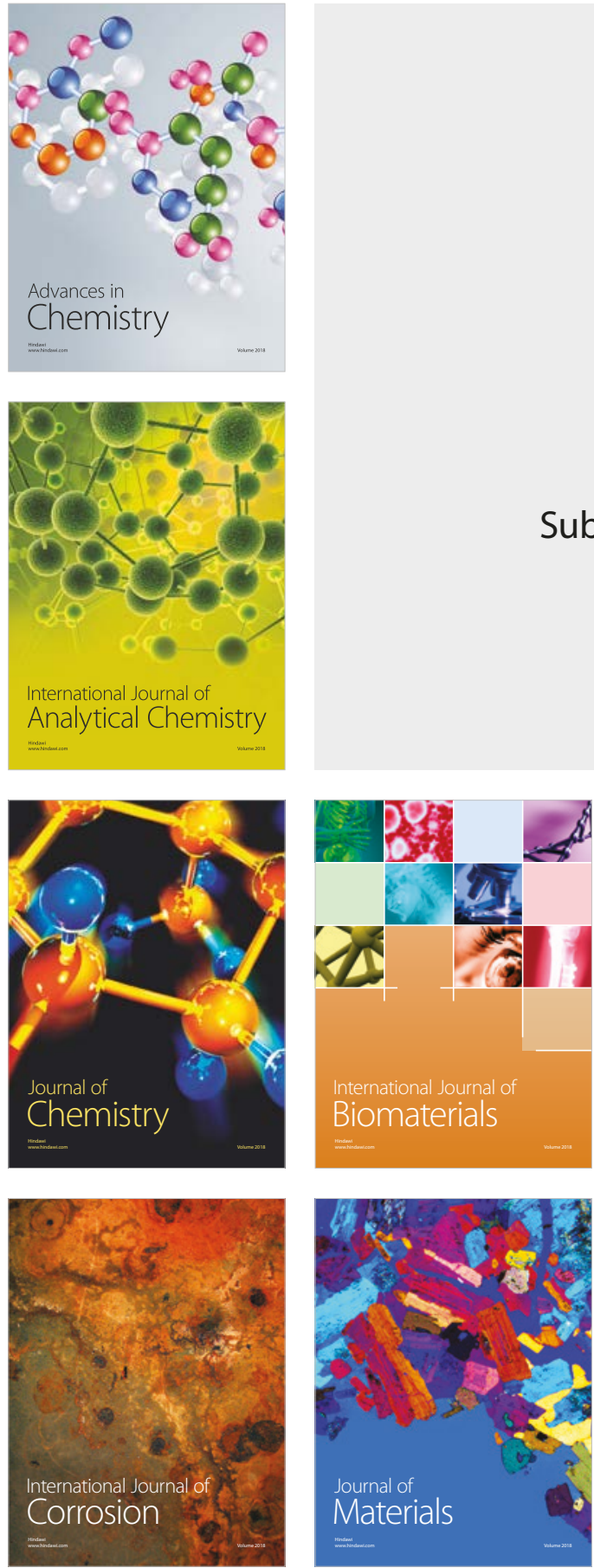

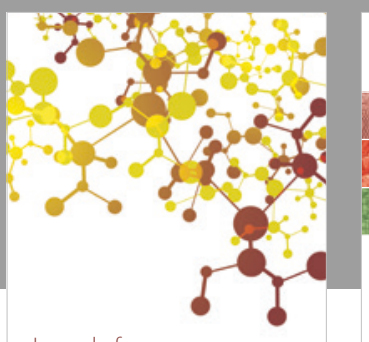

Journal of

Applied Chemistry
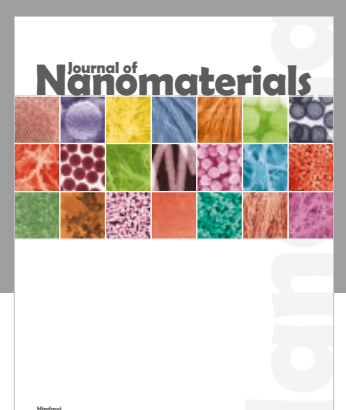

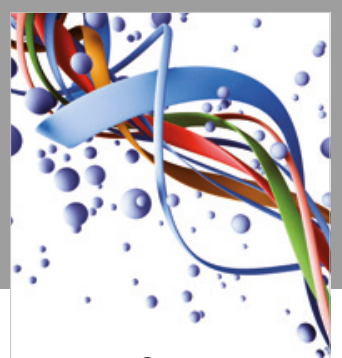

Scientifica

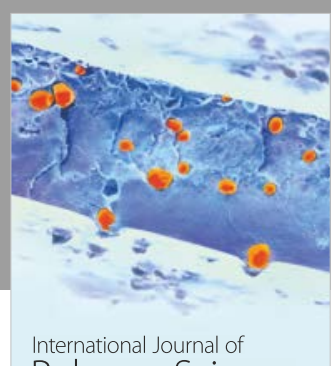

Polymer Science

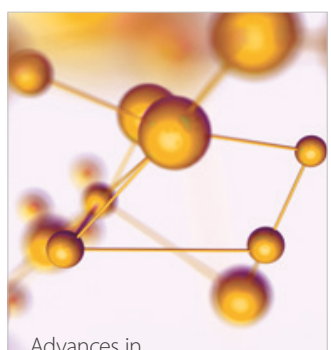

Physical Chemistry
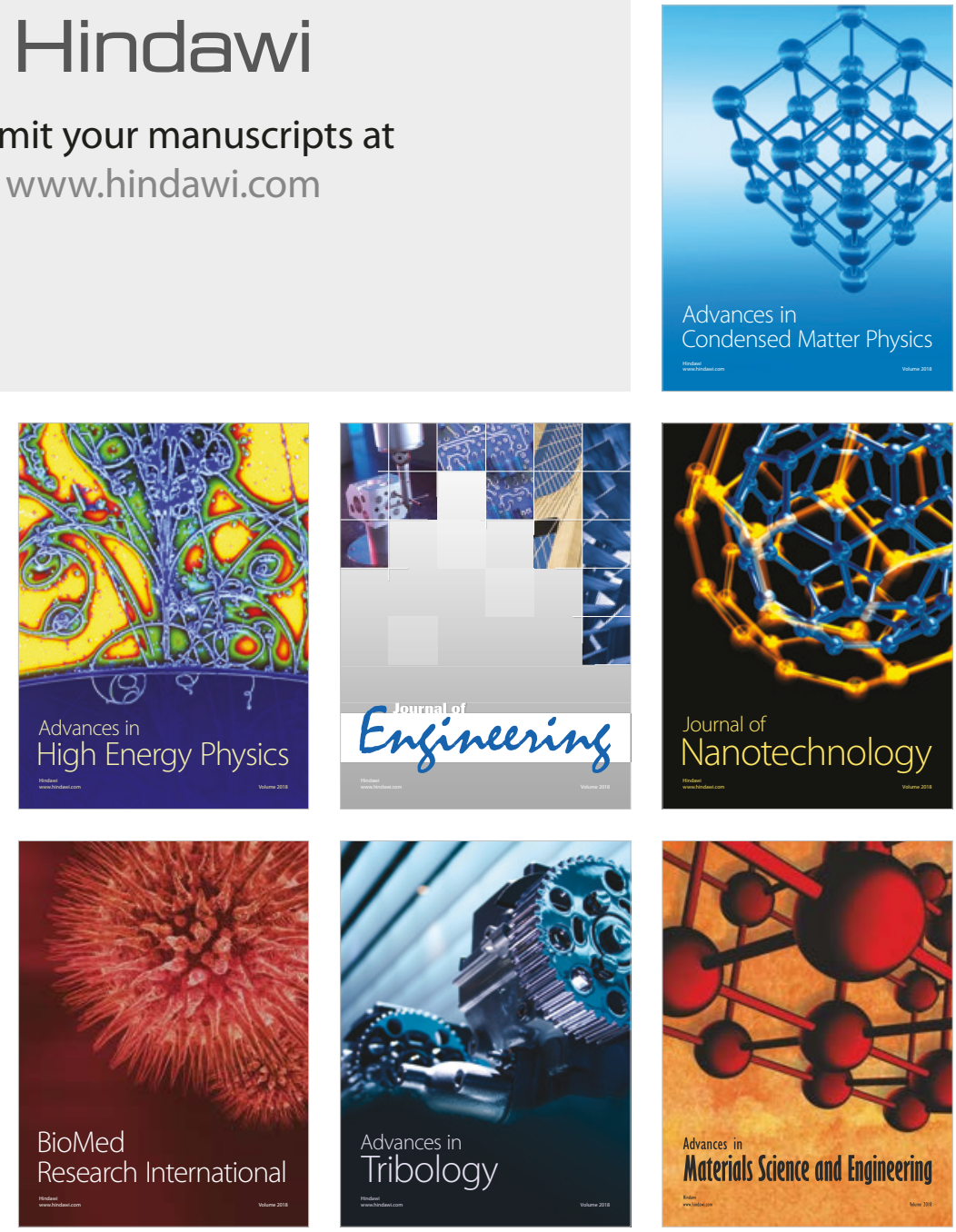